\title{
Effects of Individualized Treadmill Endurance Training on Oxidative Stress in Skeletal Muscles of Transgenic Sickle Mice
}

\author{
Etienne Gouraud, ${ }^{1,2}$ Emmanuelle Charrin, ${ }^{1,2,3}$ John J. Dubé, ${ }^{4,5}$ Solomon F. Ofori-Acquah, ${ }^{3,6}$ \\ Cyril Martin (D), ${ }^{1,2}$ Sarah Skinner, ${ }^{1,2}$ Benjamin Chatel, ${ }^{7}$ Anaelle Boreau, ${ }^{1,2}$

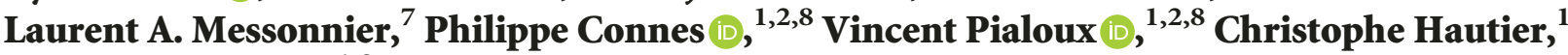 \\ and Camille Faes $\mathbb{D}^{1,2}$ \\ ${ }^{1}$ Interuniversity Laboratory of Human Movement Biology EA7424, Vascular Biology and Red Blood Cell Team, University Claude \\ Bernard Lyon 1, Villeurbanne, France \\ ${ }^{2}$ Laboratory of Excellence "GR-Ex", Paris, France \\ ${ }^{3}$ Division of Hematology/Oncology, Department of Medicine, University of Pittsburgh, Pittsburgh, PA 15261, USA \\ ${ }^{4}$ Division of Endocrinology and Metabolism, University of Pittsburgh, Pittsburgh, PA 15261, USA \\ ${ }^{5}$ Department of Biology, Chatham University, Pittsburgh, PA 15232, USA \\ ${ }^{6}$ Center for Translational and International Hematology, Vascular Medicine Institute, University of Pittsburgh, Pittsburgh, \\ PA 15261, USA \\ ${ }^{7}$ Interuniversity Laboratory of Human Movement Biology EA7424, Vascular Biology and Red Blood Cell Team, University Savoie \\ Mont Blanc, Chambéry, France \\ ${ }^{8}$ Institut Universitaire de France, Paris, France
}

Correspondence should be addressed to Camille Faes; camille.faes@univ-lyon1.fr

Received 28 November 2018; Revised 1 March 2019; Accepted 23 June 2019; Published 24 July 2019

Academic Editor: Cristina Angeloni

Copyright (c) 2019 Etienne Gouraud et al. This is an open access article distributed under the Creative Commons Attribution License, which permits unrestricted use, distribution, and reproduction in any medium, provided the original work is properly cited.

Oxidative stress is a key feature in the pathophysiology of sickle cell disease. Endurance training has been shown to reduce oxidative stress in the heart and the liver of sickle mice. However, the effects of endurance training on skeletal muscles, which are major producers of reactive oxygen species during exercise, are currently unknown. The aim of this study was to evaluate the effect of sickle genotype on prooxidant/antioxidant response to individualized endurance training in skeletal muscles of sickle mice. Healthy and homozygous Townes sickle mice were divided into trained or sedentary groups. Maximal aerobic speed and $\mathrm{VO}_{2}$ peak were determined using an incremental test on a treadmill. Trained mice ran at $40 \%$ to $60 \%$ of maximal aerobic speed, $1 \mathrm{~h}$ /day, 5 days/week for 8 weeks. Oxidative stress markers, prooxidant/antioxidant response, and citrate synthase enzyme activities were assessed in the gastrocnemius, in the plantaris, and in the soleus muscles. Maximal aerobic speed and $\dot{\mathrm{VO}}_{2}$ peak were significantly reduced in sickle compared to healthy mice $(-57 \%$ and $-17 \% ; p<0.001)$. NADPH oxidase, superoxide dismutase, and catalase activities significantly increased after training in the gastrocnemius of sickle mice only. A similar trend was observed for citrate synthase activity in sickle mice $(p=0.06)$. In this study, we showed an adaptive response to individualized endurance training on the prooxidant/antioxidant balance in the gastrocnemius, but neither in the plantaris nor in the soleus of trained sickle mice, suggesting an effect of sickle genotype on skeletal muscle response to endurance treadmill training. 


\section{Introduction}

Sickle cell disease (SCD) is a genetic disorder characterized by hemolytic anemia and vasoocclusive crises [1] resulting in an enhanced production of reactive oxygen species (ROS) $[2,3]$. In this context, higher levels of markers of oxidative stress have been found in the plasma and RBC of patients with SCD [4], as well as in the liver, kidney, and spleen of transgenic sickle mice [5].

Little research has been conducted to understand the pathophysiology of skeletal muscles in SCD, but recently, several studies started to report functional and histological alterations in skeletal muscle [6-9]. In SCD patients at rest, Ravelojaona et al. [8] observed skeletal muscle microvasculature remodeling, amyotrophy, and decreased activity of key enzymes involved in energy metabolism, including creatine kinase (CK), which could be explained by an excessive ROS content in the skeletal muscle of SCD patients [10]. Additionally, recent studies suggest that increased oxidative stress may impair force production in transgenic sickle mice during acute exercise [6] which could be attributed to the increased intramyocellular acidosis [11, 12] observed in these mice [7]. However, while it is well recognized that skeletal muscle is the major endogenous source of ROS during exercise [13, 14], little research has been done to investigate oxidative stress production in the skeletal muscle of homozygous SCD patients or mice.

While vigorous physical activity is usually not recommended for SCD patients [15], recently, a randomized controlled trial reported that an individualized and standardized moderate-intensity training program in sickle patients was clinically safe and improved the functional capacity and the skeletal muscle characteristics of those patients [16]. Besides, chronic exercise in two transgenic sickle mice models $[17,18]$ was reported to decrease oxidative stress and inflammation in several organs. In SAD mice, voluntary wheel running protocol decreased lipid peroxidation in the heart after hypoxia/reoxygenation stress [17]. In Townes mice, an endurance treadmill running protocol decreased oxidative stress in the heart and the liver and attenuated systemic inflammation [18]. However, Chatel et al. [19] reported that such training did not induce muscular oxidative stress in Townes mice. Nevertheless, the running speed was not individualized for each mouse that may explain the lack of significant changes in Chatel et al.'s study. From a clinical point of view, the individualization of the exercise in sickle cell disease patients is of primary importance in the training management of these patients.

Thus, we chose to investigate the effects of 8 -week individualized moderate-intensity treadmill training program on the oxidative stress levels in the skeletal muscle of healthy and transgenic Townes sickle mice. The main hypothesis of our work is that sickle genotype affects the prooxidant/antioxidant response in the skeletal muscle of Townes mice subjected to such endurance training program. We chose the gastrocnemius, the plantaris, and the soleus in this study for their specific typology and involvement in treadmill locomotion.

\section{Material and Methods}

2.1. Mice. The study was approved by the Institutional Animal Care and Use Committee (IACUC) at the University of Pittsburgh (protocol \#13102567). Eight-week-old male Townes mice were used in this study. Townes mice were obtained by establishing a colony using breeding pairs purchased from Jackson Laboratory (Bar Harbor, ME, USA). Mice were provided with food and water ad libitum and maintained on a 12-hour light-dark cycle.

2.2. Incremental Treadmill Test. Healthy AA $(n=12)$ and sickle SS $(n=17)$ mice were progressively acclimatized to treadmill exposure by increasing intensity and duration for 3 days. For the incremental test, mice ran on an enclosed, single lane treadmill (molecular enclosed metabolic treadmill for mice; Columbus Instruments), and real-time measurements of oxygen consumption $\left(\dot{\mathrm{VO}}_{2}\right)$ and carbon dioxide output $\left(\dot{\mathrm{V} C \mathrm{C}_{2}}\right)$ were performed using an Oxymax/Comprehensive Laboratory Animal Monitoring System (CLAMS; Columbus Instruments). Mice ran at 5, 9, 12, and $15 \mathrm{~m} \cdot \mathrm{min}^{-1}$ at a $15^{\circ}$ inclination for $5 \mathrm{~min}$ at each velocity [20]. Treadmill velocity was then increased by $2 \mathrm{~m} \cdot \mathrm{min}^{-1}$ every $2 \mathrm{~min}$ until exhaustion, defined as the inability to return to treadmill running after 10 seconds. Maximal aerobic speed (MAS) was defined as the speed at which $\dot{\mathrm{VO}}_{2}$ plateaued. We chose to do not assess $\dot{\mathrm{VO}}_{2}$ posttraining to keep enough mice in each group of mice and to avoid bias analysis. Indeed, the risk of death of the Townes mice during this kind of incremental maximal exercise test dramatically increases with age according to the progression of disease severity [21].

\subsection{Individualized Treadmill Aerobic Training Protocol.} After 7 days of rest, they were randomly distributed into 4 groups: trained AA (Tr-AA), sedentary AA (Sed-AA), trained SS (Tr-SS), and sedentary SS (Sed-SS). Trained mice ran $1 \mathrm{~h} /$ day, 5 days/week at 40\%-60\% MAS for 8 weeks at $15^{\circ}$ inclination on a motorized treadmill (Figure 1) [22] (RM Exer-3/6 open treadmill with manual incline; Columbus Instruments). If mice did not keep up with treadmill speed, they were manually encouraged to run and/or exposed to brief periods of electric shock. The sedentary groups were exposed to the treadmill 1 day/week and handled daily in the same way as mice in the trained group.

2.4. Muscle Sampling. Mice were anesthetized and euthanized by cervical dislocation $72 \mathrm{~h}$ after the last exercise session. The gastrocnemius, soleus, and plantaris were harvested, weighed, and then immediately frozen in liquid nitrogen. Muscles were homogenized in a lysis buffer $(20 \mathrm{mM}$ Tris; $1 \mathrm{mM}$ ethylenediaminetetraacetic acid; $100 \mathrm{mM}$ sodium chloride; $0.5 \%$ $(v / v)$ Triton $\mathrm{x} 100)$ and centrifuged at $12000 \mathrm{RCF}$ and $4^{\circ} \mathrm{C}$ for $10 \mathrm{~min}$. Protein concentrations were assayed with a BCA kit (Novagen \#71285.3, Darmstadt, Germany), and all results were normalized by grams of total protein.

2.5. Oxidative Stress and Antioxidant Enzyme Activities in Skeletal Muscle. All biochemical products used for oxidative stress assays were purchased from Sigma-Aldrich (St. Louis, 


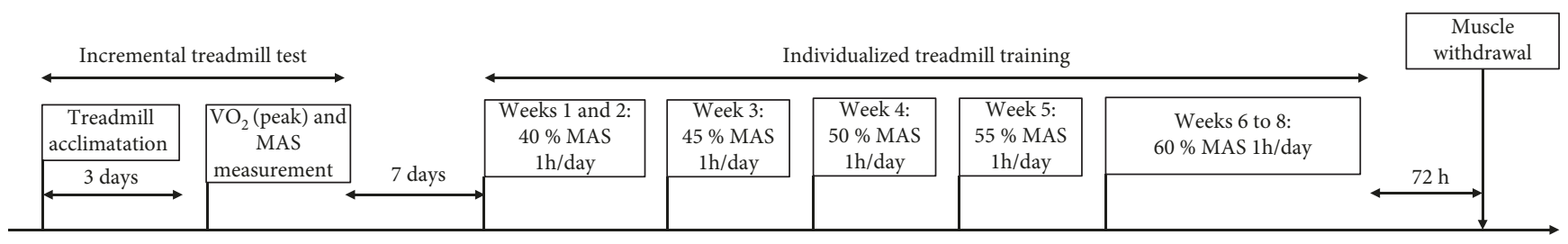

FIGURE 1: Schematic view of the training protocol procedure for healthy and sickle trained mice. $\dot{V O}_{2}$ (peak): peak oxygen consumption; MAS: maximal aerobic speed.

MO, USA), and spectrophotometric measurements were performed on a TECAN Infinite 2000 plate reader (Männedorf, Switzerland).

2.5.1. Oxidative Stress Markers. Muscle malondialdehyde (MDA) concentrations were determined as thiobarbituric reactive substances, as previously described [23]. NADPH oxidase (NOX) and xanthine oxidase (XO) activities were calculated by measuring the kinetic of appearance of the complex superoxide anion/nitrotetrazolium blue (NTB) spectrophotometrically at $560 \mathrm{~nm}$ [24].

2.5.2. Antioxidant Enzymes. Glutathione peroxidase (GPX) activity in skeletal muscle was assayed using Paglia and Valentine's [25] modified method, which uses hydrogen peroxide $\left(\mathrm{H}_{2} \mathrm{O}_{2}\right)$ as a substrate. Superoxide dismutase (SOD) activity was quantified using the Beauchamp and Fridovich's [26] method, slightly modified by Oberley and Spitz [27]. Catalase (Cat) activity in the skeletal muscle was determined using Johansson and Borg's [28] method, which uses $\mathrm{H}_{2} \mathrm{O}_{2}$ as a substrate and formaldehyde as a standard.

2.5.3. Citrate Synthase Activity. Citrate synthase (CS) activity was assessed following the production of mercaptide ions spectrophotometrically at $412 \mathrm{~nm}$ by adding dinitrothiocyanobenzene (DTNB), acetyl-CoA, and oxaloacetic acid.

2.6. Statistical Analysis. Data are expressed as the mean \pm SEM. Statistical analyses were performed using GraphPad Prism 6 (GraphPad Software, La Jolla, CA, USA). Normality was checked using the Kolmogorov-Smirnov test. Student's $t$-test was used to compare exercise capacity between healthy and sickle mice. Two-way ANOVA followed by Tukey's post hoc test was used to compare enzyme activities and oxidative stress markers among groups. The significance level was set at $p<0.05$. A tendency was also considered for $0.05 \leq p<0.1$. The statistical power $(\beta)$ has been calculated with and alpha level set at 0.05 .

\section{Results}

3.1. Peak Exercise Capacity. At baseline, incremental treadmill tests showed that SS mice had $17 \%$ lower $\mathrm{VO}_{2}$ peak than AA mice (Table 1). Also, MAS was significantly decreased in SS mice compared to AA mice (-57\%; Table 1$)$.

3.2. Citrate Synthase Activity. In the gastrocnemius, CS activity was significantly higher in SS compared to AA mice in the trained group $(p<0.05, \beta=0.92)$. It tended to be higher in trained SS compared to their sedentary counterparts
TABle 1: Peak exercise capacity activity for all groups prior to training.

\begin{tabular}{lcc}
\hline & $\mathrm{AA}(n=12)$ & $\mathrm{SS}(n=17)$ \\
\hline $\mathrm{VO}_{2}$ peak $\left(\mathrm{ml} \cdot \mathrm{h}^{-1} \cdot \mathrm{kg}^{-1}\right)$ & $8407.0 \pm 224.5$ & $7046 \pm 118.2^{* * *}$ \\
$\begin{array}{l}\text { Maximal aerobic } \\
\text { speed }\left(\mathrm{m} \cdot \mathrm{min}^{-1}\right)\end{array}$ & $29.3 \pm 1.7$ & $13.6 \pm 0.5^{* * *}$ \\
\hline
\end{tabular}

Data are expressed as mean \pm SEM. ${ }^{* * *} p<0.001$.

$(p=0.06, \beta=0.81$, Figure $2(a))$ while no differences were observed between Tr-AA and Sed-AA mice $(p=0.99$, $\beta=0.06$, Figure 2(a)).

In the plantaris and the soleus, CS activity did not differ between the four groups.

3.3. Prooxidant and Oxidative Stress Markers. In the gastrocnemius, a significant interaction effect (genotype $\times$ training, $p<0.01, \beta=0.99$ ) was observed for NOX while neither genotype nor training effects were identified for XO activity (Figures 2(b) and 2(c)). NOX activity was 2-fold higher $(p<0.001, \beta=1)$ in trained SS compared to their sedentary counterparts (Figure 2(b)). Training did not significantly change MDA in healthy or sickle mice. However, a significant genotype effect $(p<0.01)$ was observed for MDA with a higher level of MDA observed in SS compared to AA mice, as evidenced by an almost twofold higher level in Tr-SS compared with Tr-AA mice (Figure 2(d)).

In the plantaris, $\mathrm{XO}$ and NOX activities were not modified by training in the AA or SS mice (Figures 3(b) and $3(\mathrm{c})$ ). In contrast, MDA concentrations were significantly higher in Tr-SS vs. Tr-AA mice $(p<0.05, \beta=1$, Figure $3(\mathrm{~d})$ ) and were twofold lower in trained $v$ s. sedentary AA mice.

In the soleus, MDA concentrations did not differ between the four groups. However, similar to the plantaris, a significant interaction effect (genotype $\times$ training, $p<0.01$, $\beta=0.72$ ) was observed with a nearly twofold higher level in Tr-SS compared to Tr-AA mice (Figure 4(c)).

3.4. Antioxidant Enzyme Activities. In the gastrocnemius of SS mice, we found significantly higher SOD $(p<0.05$, $\beta=0.96$, Figure $2(\mathrm{e}))$ and catalase activities $(p<0.05$, $\beta=0.82$, Figure $2(\mathrm{f}))$ ( $50 \%$ and $85 \%$, respectively) in $\mathrm{Tr}$-SS compared to Sed-SS mice. In addition, significantly higher SOD activity was found in Tr-SS compared to Tr-AA mice $(p<0.05, \beta=0.99$, Figure 2(e)). Training did not significantly affect GPX activity in Tr-SS compared to Sed-SS mice $(p=0.12, \beta=0.57$, Figure $2(\mathrm{~g}))$. However, a genotype effect 


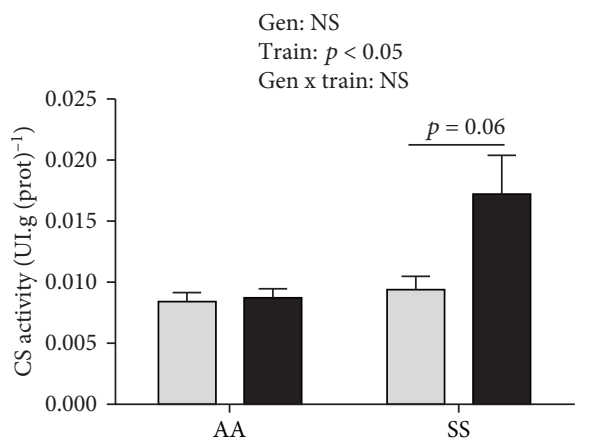

(a)

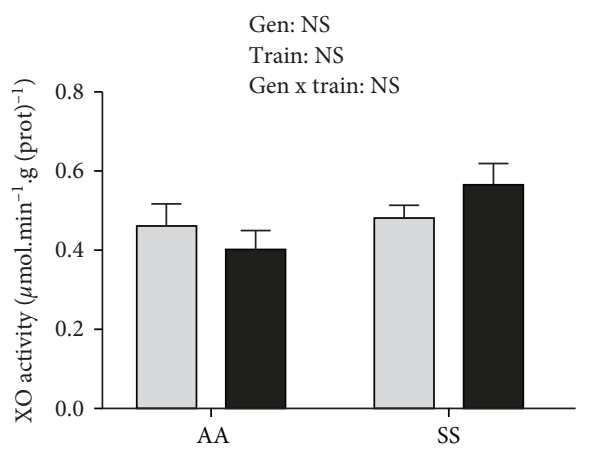

(c)

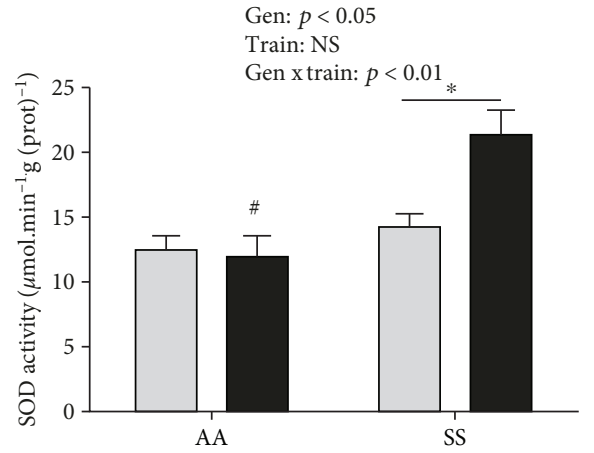

(e)

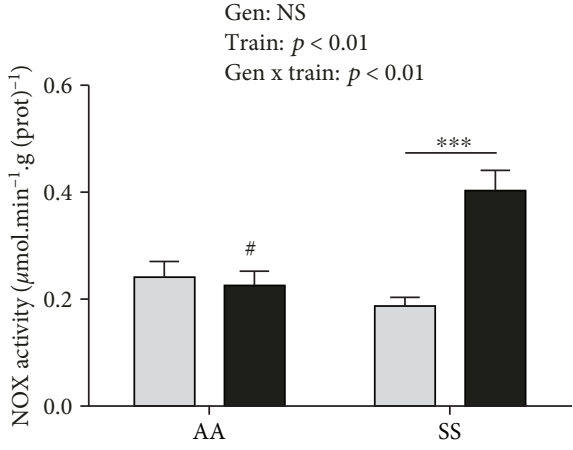

(b)

Gen: $p<0.01$

Train: NS

Gen $\mathrm{x}$ train: NS

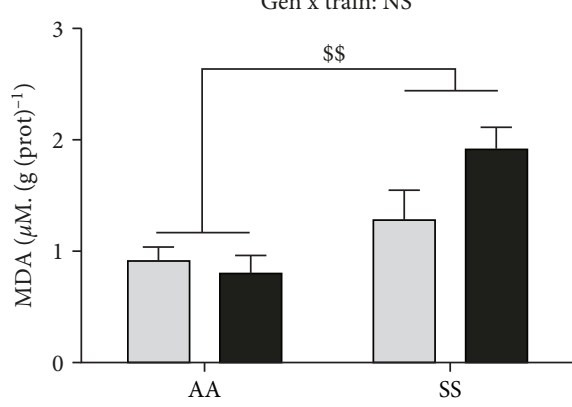

(d)

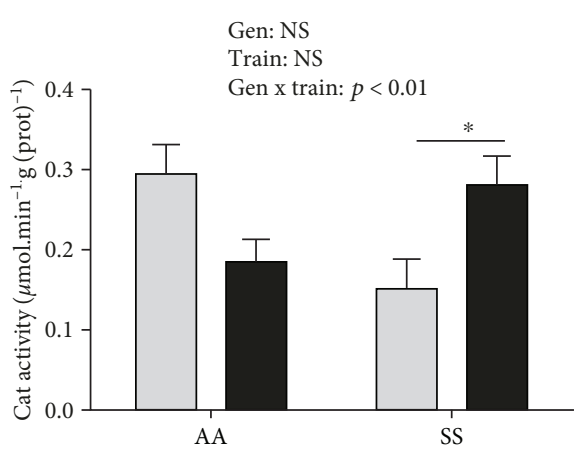

(f)

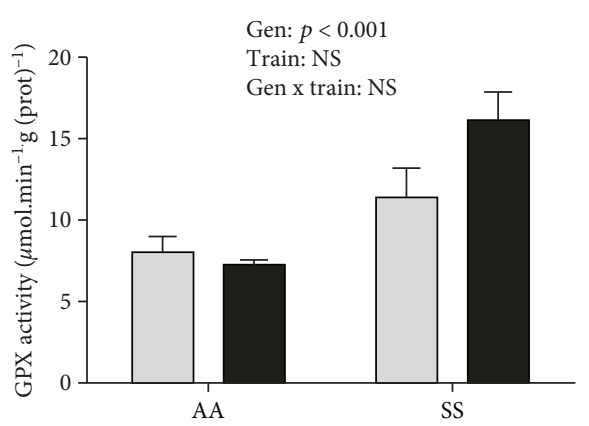

(g)

Figure 2: Citrate synthase (CS) activity (a), prooxidant enzyme activities (b, c) malondialdehyde (MDA) concentration (d), and antioxidant enzyme activities (e-g) in the gastrocnemius. Cat: catalase; GPX: glutathione peroxidase; NOX: NADPH oxidase; SOD: superoxide dismutase; $\mathrm{XO}$ : xanthine oxidase. Data are expressed as the mean \pm SEM. Gen: genotype effect; Train: training effect; Gen $\mathrm{x}$ train: genotype $\times$ interaction effect. ${ }^{*} p<0.05,{ }^{* * *} p<0.001,{ }^{\$} p<0.01$, and ${ }^{*} p<0.01 v$ s. Tr-SS. $n$ (Sed-AA $)=6, n(\operatorname{Tr}-\mathrm{AA})=6, n($ Sed-SS $)=7$, and $n($ Tr-SS $)=$ 10. Grey bars: sedentary mice; black bars: trained mice. 


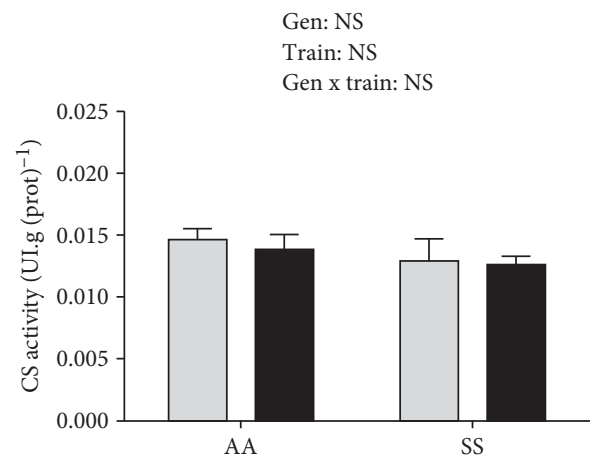

(a)

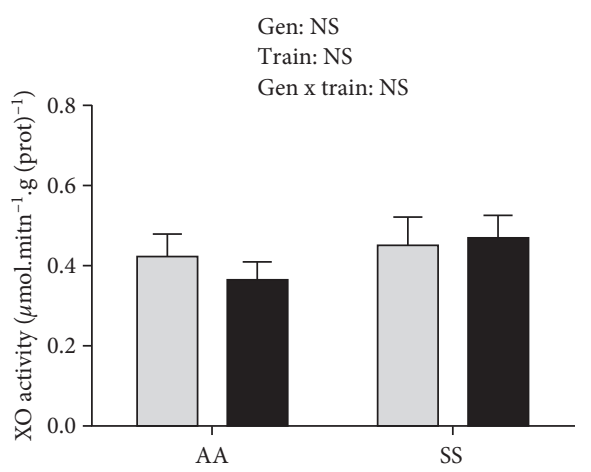

(c)

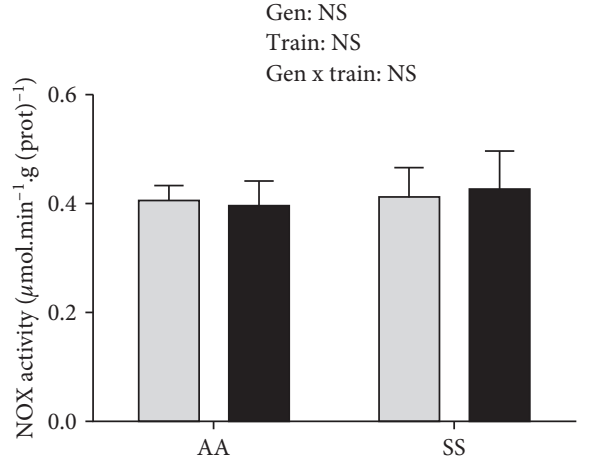

(b)

Gen: $p<0.05$

Train: NS

Gen $x$ train: $p<0.05$

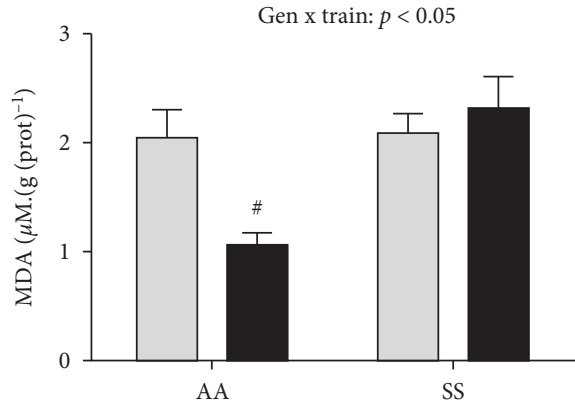

(d)

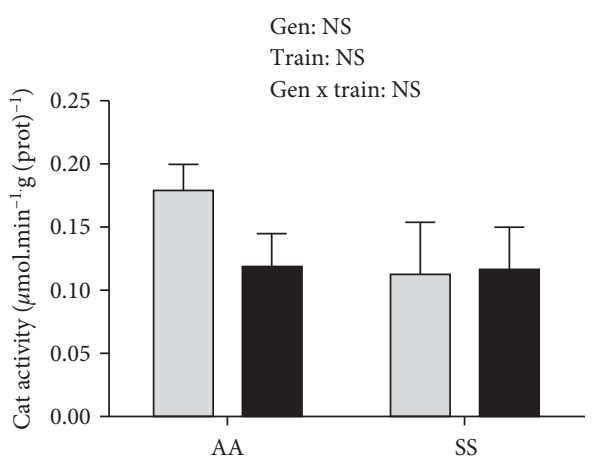

(f)

(e)

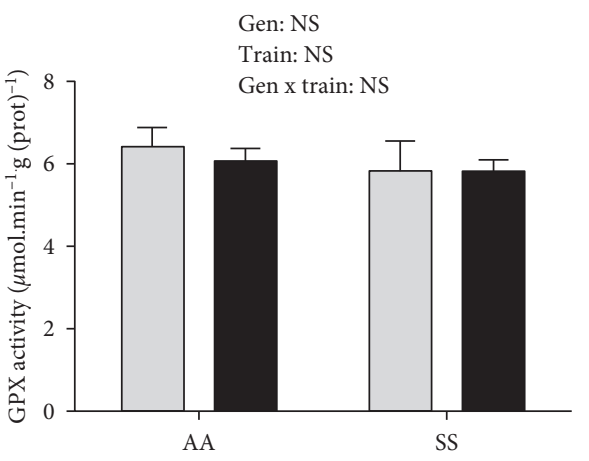

(g)

FIGURE 3: Citrate synthase (CS) activity (a), prooxidant enzyme activities (b, c) malondialdehyde (MDA) concentration (d), and antioxidant enzyme activities (e-g) in the plantaris. Cat: catalase; GPX: glutathione peroxidase; NOX: NADPH oxidase; SOD: superoxide dismutase; XO: xanthine oxidase. Data are expressed as the mean \pm SEM. Gen: genotype effect; Train: training effect; Gen $x$ train: genotype $\times$ interaction effect. ${ }^{\#} p<0.05 v$ s. Tr-SS. $n($ Sed-AA $)=6, n(\operatorname{Tr}-\mathrm{AA})=6, n($ Sed-SS $)=7$, and $n(\mathrm{Tr}-\mathrm{SS})=10$. Grey bars: sedentary mice; black bars: trained mice. 


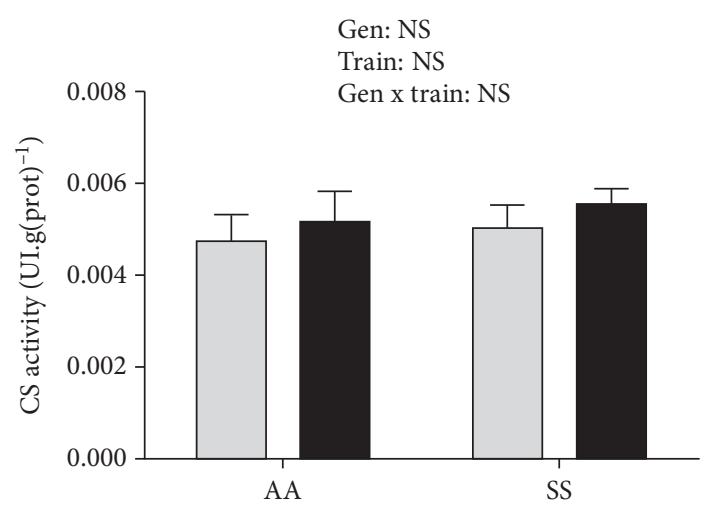

(a)

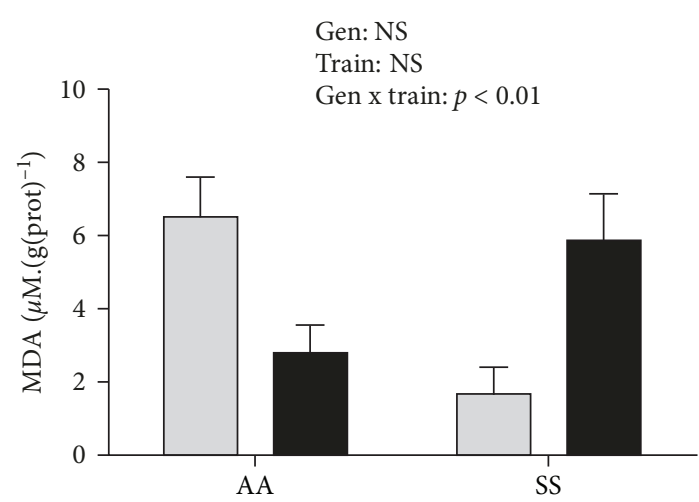

(c)

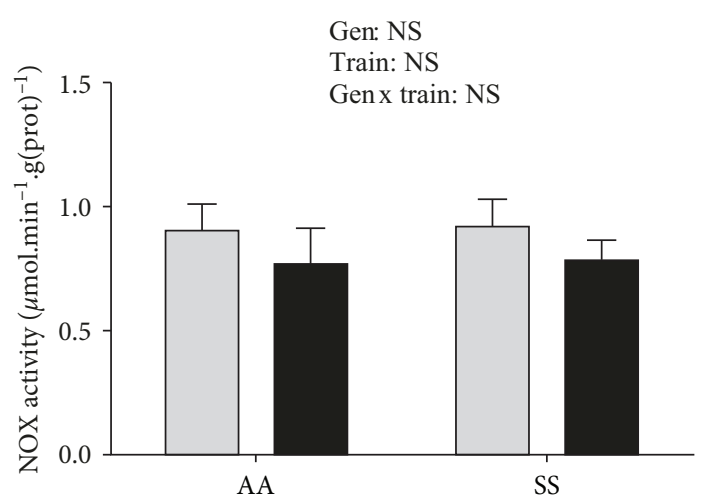

(b)

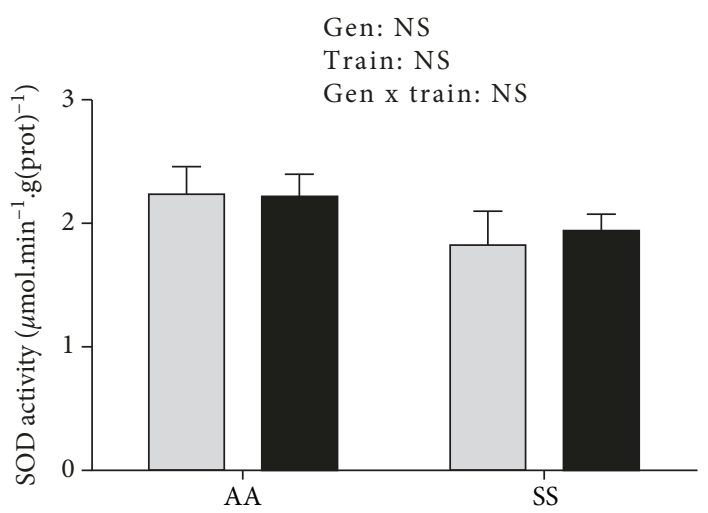

(d)

FIGURE 4: Citrate synthase (CS) activity (a), NADPH oxidase (NOX) activity (b), malondialdehyde (MDA) concentration (c), and superoxide dismutase (SOD) activity (d) in the soleus. Data are expressed as the mean \pm SEM. Gen: genotype effect; Train: training effect; Gen $x$ train: genotype $\times$ interaction effect. $n($ Sed-AA $)=6, n(\operatorname{Tr}-\mathrm{AA})=6, n($ Sed-SS $)=7$, and $n(\operatorname{Tr}-\mathrm{SS})=10$. Grey bars: sedentary mice; black bars: trained mice.

was identified $(p<0.01)$ with higher GPX in SS compared to AA mice independent of a sedentary or trained status.

In the plantaris and in the soleus, neither training nor genotype effects were observed (Figures 3(e)-3(g); Figure 4(d)).

\section{Discussion}

The aim of this study was to evaluate the effects of sickle genotype on the prooxidant/antioxidant response to endurance exercise in three skeletal muscles of SCD transgenic mice. Our results demonstrated that an 8-week individualized moderate-intensity treadmill training program (i) increased oxidative stress markers and (ii) increased antioxidant enzyme activity in the gastrocnemius, but not in the plantaris and in the soleus of trained Townes SS mice.

Markers of lipid peroxidation and prooxidant enzymes activities are higher in SS mice after 8 weeks of individualized moderate-intensity treadmill training program. Indeed, NOX [29] was reported as a main producer of ROS in skeletal muscle, and CS [30] has been extensively used as a marker for assessing muscle mitochondrial volume density as well as mitochondrial ROS production. Both may indicate higher ROS production in the gastrocnemius. Also, elevated intracellular acidosis previously observed in these mice [7] may cause higher ROS production. Acidosis has been shown to increase oxidative stress by promoting Fenton reactions and by causing a protonation of the peroxynitrite anion that can lead to the production of radical hydroxyl, one of the most powerful ROS [11, 12]. Meanwhile, the 8-week individualized moderate-intensity treadmill training program also increased antioxidant enzymes. This suggests that the proantioxidant balance in the gastrocnemius of trained sickle mice could be equilibrated as illustrated by the lack of change in MDA in response to the exercise training. Our findings are in accordance with previous studies in rodents after aerobic training [31-33], as we found higher SOD and catalase activities in trained sickle mice. This increase of antioxidant enzyme activities could be attributed to the increase in NOX activity and subsequent ROS production involved in the oxidative stress signaling pathway $[33,34]$. Higher CS activity may also be explained by an increased ROS production which could act as a signaling stimulus to increase mitochondrial content and oxidative capacity in skeletal muscles [35]. As previously reported in healthy mice, exogenous supplementation of $\mathrm{N}$-acetylcysteine, a precursor of glutathione synthesis, was found to attenuate exercise-induced upregulation of endogenous antioxidants, including SOD and catalase activities in the gastrocnemius [33]. Those results suggest [33] the importance of a minimal ROS content 
(i.e., mild prooxidant environment) to training-induced adaptation of skeletal muscle.

While endurance training has been found to reduce inflammation, splenic enlargement, cardiac and hepatic oxidative stress in a similar transgenic sickle mice model [18], these results suggest an adaptive response to endurance training on pro-antioxidant balance in the skeletal muscle of sickle mice. Surprisingly, resting NOX and XO activities as well as MDA concentration did not differ between AA and SS sedentary mice. Therefore, it is likely that oxidative stress is not changed at rest in skeletal muscle in sedentary sickle mice, unlike in their trained counterparts. This could also suggest that skeletal muscle may be less affected by the disease than other organs in these mice [5].

Changes in the balance of pro-antioxidant markers in the gastrocnemius after an 8-week individualized moderateintensity treadmill training program were only observed in trained SS mice and not in their AA counterparts. Previous results reported no modifications in muscular oxidative stress in AA mice exposed to endurance training program [19] while higher training intensity was shown to decrease oxidative stress and increase antioxidant markers in the muscle of healthy mice [36]. Therefore, our training intensity/speed (40\%-60\% MAS) could not be enough to trigger oxidative stress adaptations in the gastrocnemius of AA Townes mice. In addition, our results in the gastrocnemius differ from those of Chatel et al. [19], who did not report changes in the pro-antioxidant balance between trained and sedentary sickle mice in the tibialis anterior after 7 weeks of aerobic training. These differences may be explained by the individualization of the training speed in our study (speed calculated from the percentage of MAS from each mice) compared to Chatel et al. [19] who used the same speed for all the mice or by the muscle investigated (i.e., tibialis anterior), whose typology is nearly similar to the plantaris [37].

While moderate endurance training induced oxidative stress adaptations in the gastrocnemius of Tr-SS, no effects were observed in the plantaris and the soleus. The differential response to endurance training in each muscle could be explained by their relative contribution in treadmill locomotion. Indeed, the gastrocnemius, which represents more than $80 \%$ of the posterior hindlimb muscles in the mice [6], has a higher contribution in the generation of mechanical work during locomotion than the plantaris and soleus [38].

Thus, it seems that the skeletal muscle work generated by running at $40-60 \%$ of MAS was sufficient to induce muscular changes in oxidative stress in Tr-SS but was too mild to trigger oxidative stress adaptations in Tr-AA mice. Therefore, our data provide evidence that the use of individualized training intensities might be a factor to consider when designing endurance training protocols for sickle cell mice or patients.

\section{Conclusion}

In conclusion, our results show that an 8-week individualized moderate-intensity treadmill training program increased prooxidant and antioxidant enzyme activities in the gastrocnemius of transgenic sickle mice suggesting an effect of SS genotype on pro-antioxidant response to endurance training in these mice. Individualization of exercise intervention should be considered in the context of therapeutic care of SCD patients, in line with the conclusions of the first randomized controlled trial training program performed in homozygous SCD patients [16]. Our results combined with the improvement in skeletal muscle characteristics reported in Gellen et al.'s study [16] strengthen the idea that skeletal muscle is a key target to consider in further therapeutic protocol. Further studies should be conducted to better characterize the percentage of training intensity that may trigger oxidative stress and skeletal muscle adaptations without associated complications in these individuals.

\section{Abbreviations}

AA: $\quad$ Healthy genotype of hemoglobin

ANOVA: Analysis of variance

AS: $\quad$ Heterozygous genotype of sickle cell disease

Cat: $\quad$ Catalase

CK: $\quad$ Creatine kinase

COX: Cytochrome $\mathrm{C}$ oxidase

CS: $\quad$ Citrate synthase

DTNB: Dinitrothiocyanobenzene

GR: Glutathione reductase

GSH: $\quad$ Glutathione

GPX: Glutathione peroxidase

$\mathrm{H}_{2} \mathrm{O}_{2}$ : Hydrogen peroxide

HbS: Hemoglobin S

MAS: Maximal aerobic speed

MDA: Malondialdehyde

NADPH: Nicotinamide adenine dinucleotide phosphate

NOX: NADPH oxidase

RBC: $\quad$ Red blood cell

ROS: Reactive oxygen species

SCD: $\quad$ Sickle cell disease

Sed-AA: Sedentary healthy Townes mice

Sed-SS: Sedentary homozygous sickle Townes mice

SOD: $\quad$ Superoxide dismutase

SS: $\quad$ Homozygous genotype of sickle cell disease

Tr-AA: Trained healthy Townes mice

Tr-SS: Trained homozygous sickle Townes mice

$\mathrm{VCO}_{2}$ : Carbon dioxide output

$\mathrm{V}_{2}: \quad$ Oxygen consumption

$\mathrm{XO} \quad$ Xanthine oxidase.

\section{Data Availability}

The data used to support the findings of this study are available from the corresponding author upon request.

\section{Conflicts of Interest}

The authors have no competing interests, including specific financial interests, relationships, and/or affiliations relevant to the subject matter or materials included in this manuscript. 


\section{Authors' Contributions}

EG, EC, JD, SFOA, CM, and $\mathrm{CH}$ are responsible for the study design. $\mathrm{EG}, \mathrm{EC}$, and $\mathrm{AB}$ are assigned to the acquisition of data. EG, BC, VP, CH, and $\mathrm{CF}$ analyzed and interpreted the data. EG, EC, VP, $\mathrm{CH}$, and $\mathrm{CF}$ drafted the manuscript. BC, $\mathrm{LM}, \mathrm{CM}$, and $\mathrm{PC}$ are responsible for the critical revision of the manuscript.

\section{Acknowledgments}

This study was conducted with research funding from the Institut Universitaire de France (PC, VP) and the National Heart, Lung, and Blood Institute (SFOA: R01 HL106192 and U01 HL117721). We thank Frances Weidert and Bethany Flage for their technical support.

\section{References}

[1] D. C. Rees, T. N. Williams, and M. T. Gladwin, "Sickle-cell disease,” The Lancet, vol. 376, no. 9757, pp. 2018-2031, 2010.

[2] G. J. Kato, M. H. Steinberg, and M. T. Gladwin, "Intravascular hemolysis and the pathophysiology of sickle cell disease," The Journal of Clinical Investigation, vol. 127, no. 3, pp. 750-760, 2017.

[3] K. Szocs, "Endothelial dysfunction and reactive oxygen species production in ischemia/reperfusion and nitrate tolerance," General Physiology and Biophysics, vol. 23, no. 3, pp. 265295, 2004.

[4] E. N. Chirico, C. Faës, P. Connes, E. Canet-Soulas, C. Martin, and V. Pialoux, "Role of exercise-induced oxidative stress in sickle cell trait and disease," Sports Medicine, vol. 46, no. 5, pp. 629-639, 2016.

[5] E. Charrin, S. F. Ofori-Acquah, E. Nader et al., "Inflammatory and oxidative stress phenotypes in transgenic sickle cell mice," Blood Cells, Molecules \& Diseases, vol. 62, pp. 13-21, 2016.

[6] B. Chatel, C. Hourdé, J. Gondin et al., "Impaired muscle force production and higher fatigability in a mouse model of sickle cell disease," Blood Cells, Molecules \& Diseases, vol. 63, pp. 37-44, 2017.

[7] B. Chatel, L. A. Messonnier, C. Hourdé, C. Vilmen, M. Bernard, and D. Bendahan, "Moderate and intense muscular exercises induce marked intramyocellular metabolic acidosis in sickle cell disease mice," Journal of Applied Physiology, vol. 122, no. 5, pp. 1362-1369, 2017.

[8] M. Ravelojaona, L. Féasson, S. Oyono-Enguéllé et al., "Evidence for a profound remodeling of skeletal muscle and Its microvasculature in sickle cell anemia," The American Journal of Pathology, vol. 185, no. 5, pp. 1448-1456, 2015.

[9] K. A. Dougherty, C. Bertolaso, J. I. Schall, K. Smith-Whitley, and V. A. Stallings, "Muscle strength, power, and torque deficits in children with type SS sickle cell disease," Journal of Pediatric Hematology/Oncology, vol. 40, no. 5, pp. 348-354, 2018.

[10] S. Genet, R. Kale, and N. Baquer, "Effects of free radicals on cytosolic creatine kinase activities and protection by antioxidant enzymes and sulfhydryl compounds," Molecular and Cellular Biochemistry, vol. 210, no. 1/2, pp. 23-28, 2000.

[11] J. A. Kellum, M. Song, and J. Li, "Science review: extracellular acidosis and the immune response: clinical and physiologic implications," Critical Care, vol. 8, no. 5, pp. 331-336, 2004.
[12] W. Hassan, M. Ibrahim, and J. B. T. Rocha, "Low pH does not modulate antioxidant status of diphenyl ditelluride but exacerbates Fe (II)-induced lipid peroxidation in liver preparation," Drug and Chemical Toxicology, vol. 32, no. 4, pp. 438-442, 2009.

[13] S. K. Powers and M. J. Jackson, "Exercise-induced oxidative stress: cellular mechanisms and impact on muscle force production," Physiological Reviews, vol. 88, no. 4, pp. 1243-1276, 2008.

[14] M. J. Jackson, A. Vasilaki, and A. McArdle, "Cellular mechanisms underlying oxidative stress in human exercise," Free Radical Biology \& Medicine, vol. 98, pp. 13-17, 2016.

[15] C. Martin, V. Pialoux, C. Faes, E. Charrin, S. Skinner, and P. Connes, "Does physical activity increase or decrease the risk of sickle cell disease complications?," British Journal of Sports Medicine, vol. 52, no. 4, pp. 214-218, 2018.

[16] B. Gellen, L. A. Messonnier, F. Galactéros et al., "Moderateintensity endurance-exercise training in patients with sicklecell disease without severe chronic complications (EXDRE): an open-label randomised controlled trial," The Lancet Haematology, vol. 5, no. 11, pp. e554-e562, 2018.

[17] E. Charrin, E. Aufradet, A. Douillard et al., "Oxidative stress is decreased in physically active sickle cell SAD mice," British Journal of Haematology, vol. 168, no. 5, pp. 747-756, 2015.

[18] E. Charrin, J. J. Dubé, P. Connes et al., "Moderate exercise training decreases inflammation in transgenic sickle cell mice," Blood Cells, Molecules, and Diseases, vol. 69, pp. 4552, 2018.

[19] B. Chatel, L. A. Messonnier, Q. Barge et al., "Endurance training reduces exercise-induced acidosis and improves muscle function in a mouse model of sickle cell disease," Molecular Genetics and Metabolism, vol. 123, no. 3, pp. 400-410, 2018.

[20] O. J. Kemi, J. P. Loennechen, U. Wisløff, and Ø. Ellingsen, "Intensity-controlled treadmill running in mice: cardiac and skeletal muscle hypertrophy," Journal of Applied Physiology, vol. 93, no. 4, pp. 1301-1309, 2002.

[21] T. M. Ryan, D. J. Ciavatta, and T. M. Townes, "Knockouttransgenic mouse model of sickle cell disease," Science, vol. 278, no. 5339, pp. 873-876, 1997.

[22] M. G. Pereira, J. C. B. Ferreira, C. R. Bueno Jr. et al., "Exercise training reduces cardiac angiotensin II levels and prevents cardiac dysfunction in a genetic model of sympathetic hyperactivity-induced heart failure in mice," European Journal of Applied Physiology, vol. 105, no. 6, pp. 843-850, 2009.

[23] V. Pialoux, R. Mounier, E. Ponsot et al., "Effects of exercise and training in hypoxia on antioxidant/pro-oxidant balance," European Journal of Clinical Nutrition, vol. 60, no. 12, pp. 1345-1354, 2006.

[24] S. Laouafa, A. Ribon-Demars, F. Marcouiller et al., "Estradiol protects against cardiorespiratory dysfunctions and oxidative stress in intermittent hypoxia," Sleep, vol. 40, no. 8, 2017.

[25] D. E. Paglia and W. N. Valentine, "Studies on the quantitative and qualitative characterization of erythrocyte glutathione peroxidase," The Journal of Laboratory and Clinical Medicine, vol. 70, no. 1, pp. 158-169, 1967.

[26] C. Beauchamp and I. Fridovich, "Superoxide dismutase: improved assays and an assay applicable to acrylamide gels," Analytical Biochemistry, vol. 44, no. 1, pp. 276-287, 1971.

[27] L. W. Oberley and D. R. Spitz, "Assay of superoxide dismutase activity in tumor tissue," Methods in Enzymology, vol. 105, pp. 457-464, 1984. 
[28] L. H. Johansson and L. A. Håkan Borg, "A spectrophotometric method for determination of catalase activity in small tissue samples," Analytical Biochemistry, vol. 174, no. 1, pp. 331336, 1988.

[29] G. K. Sakellariou, M. J. Jackson, and A. Vasilaki, "Redefining the major contributors to superoxide production in contracting skeletal muscle. The role of NAD $(\mathrm{P}) \mathrm{H}$ oxidases," Free Radical Research, vol. 48, no. 1, pp. 12-29, 2013.

[30] S. Larsen, J. Nielsen, C. N. Hansen et al., "Biomarkers of mitochondrial content in skeletal muscle of healthy young human subjects," The Journal of Physiology, vol. 590, no. 14, pp. 3349-3360, 2012.

[31] S. K. Powers, D. Criswell, J. Lawler et al., "Influence of exercise and fiber type on antioxidant enzyme activity in rat skeletal muscle," American Journal of Physiology-Regulatory, Integrative and Comparative Physiology, vol. 266, no. 2, Part 2, pp. R375-R380, 1994.

[32] D. R. Plant, P. Gregorevic, S. A. Warmington, D. A. Williams, and G. S. Lynch, "Endurance training adaptations modulate the redox-force relationship of rat isolated slow-twitch skeletal muscles," Clinical and Experimental Pharmacology \& Physiology, vol. 30, no. 1-2, pp. 77-81, 2003.

[33] T. L. Merry and M. Ristow, "Nuclear factor erythroid-derived 2-like 2 (NFE2L2, Nrf2) mediates exercise-induced mitochondrial biogenesis and the anti-oxidant response in mice," The Journal of Physiology, vol. 594, no. 18, pp. 5195-5207, 2016.

[34] P. Steinbacher and P. Eckl, "Impact of oxidative stress on exercising skeletal muscle," Biomolecules, vol. 5, no. 2, pp. 356-377, 2015.

[35] N. A. Strobel, J. M. Peake, A. Matsumoto, S. A. Marsh, J. S. Coombes, and G. D. Wadley, "Antioxidant supplementation reduces skeletal muscle mitochondrial biogenesis," Medicine and Science in Sports and Exercise, vol. 43, no. 6, pp. 10171024, 2011.

[36] J. C. B. Ferreira, A. V. Bacurau, C. R. Bueno Junior et al., "Aerobic exercise training improves $\mathrm{Ca}^{2+}$ handling and redox status of skeletal muscle in mice," Experimental Biology and Medicine, vol. 235, no. 4, pp. 497-505, 2010.

[37] D. Bloemberg and J. Quadrilatero, "Rapid determination of myosin heavy chain expression in rat, mouse, and human skeletal muscle using multicolor immunofluorescence analysis," PLoS One, vol. 7, no. 4, article e35273, 2012.

[38] B. I. Prilutsky, W. Herzog, and T. L. Allinger, "Mechanical power and work of cat soleus, gastrocnemius and plantaris muscles during locomotion: possible functional significance of muscle design and force patterns," The Journal of Experimental Biology, vol. 199, Part 4, pp. 801-814, 1996. 


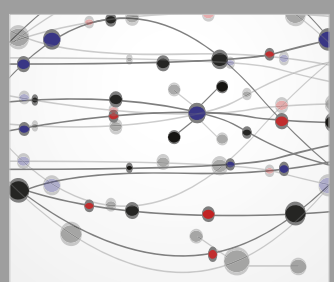

The Scientific World Journal
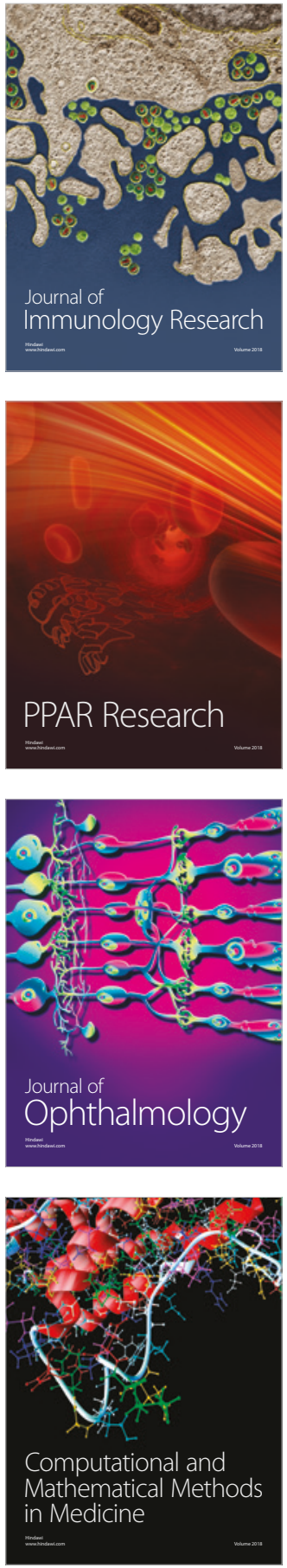

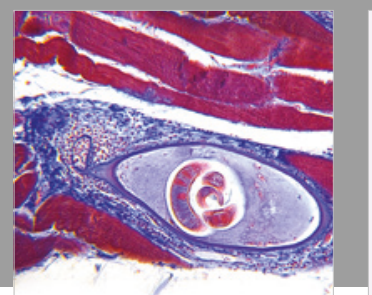

Gastroenterology Research and Practice

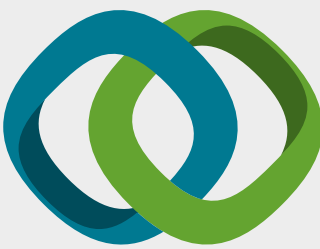

\section{Hindawi}

Submit your manuscripts at

www.hindawi.com
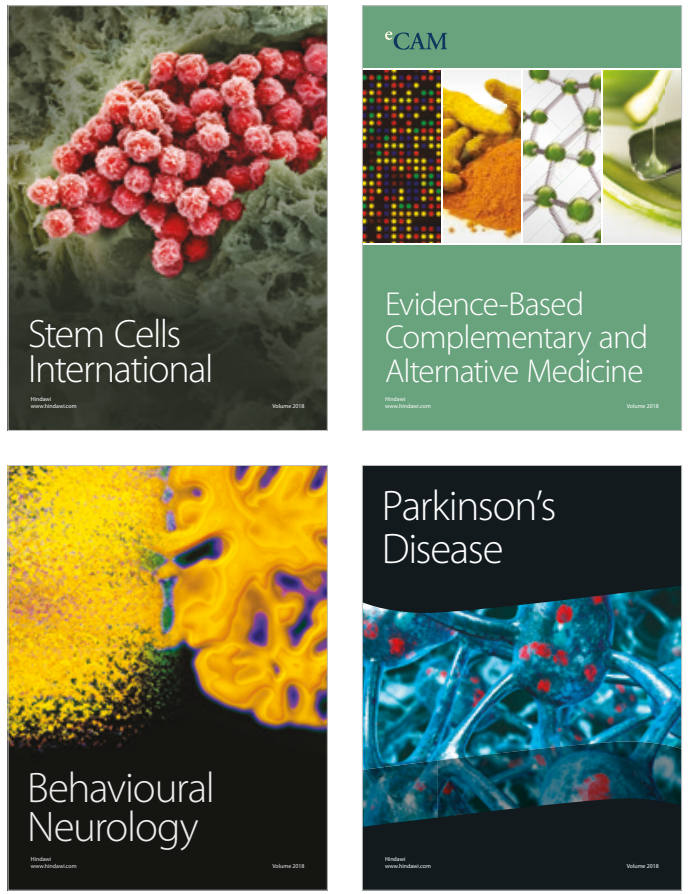

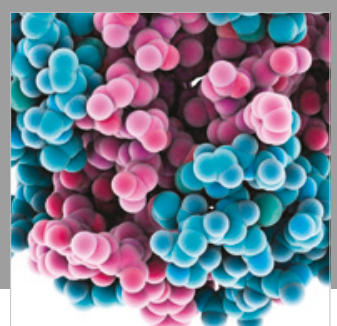

ournal of

Diabetes Research

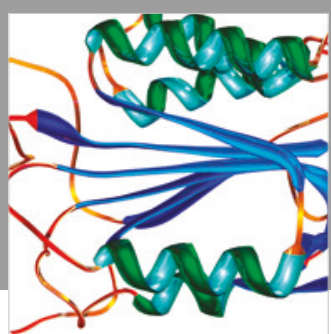

Disease Markers
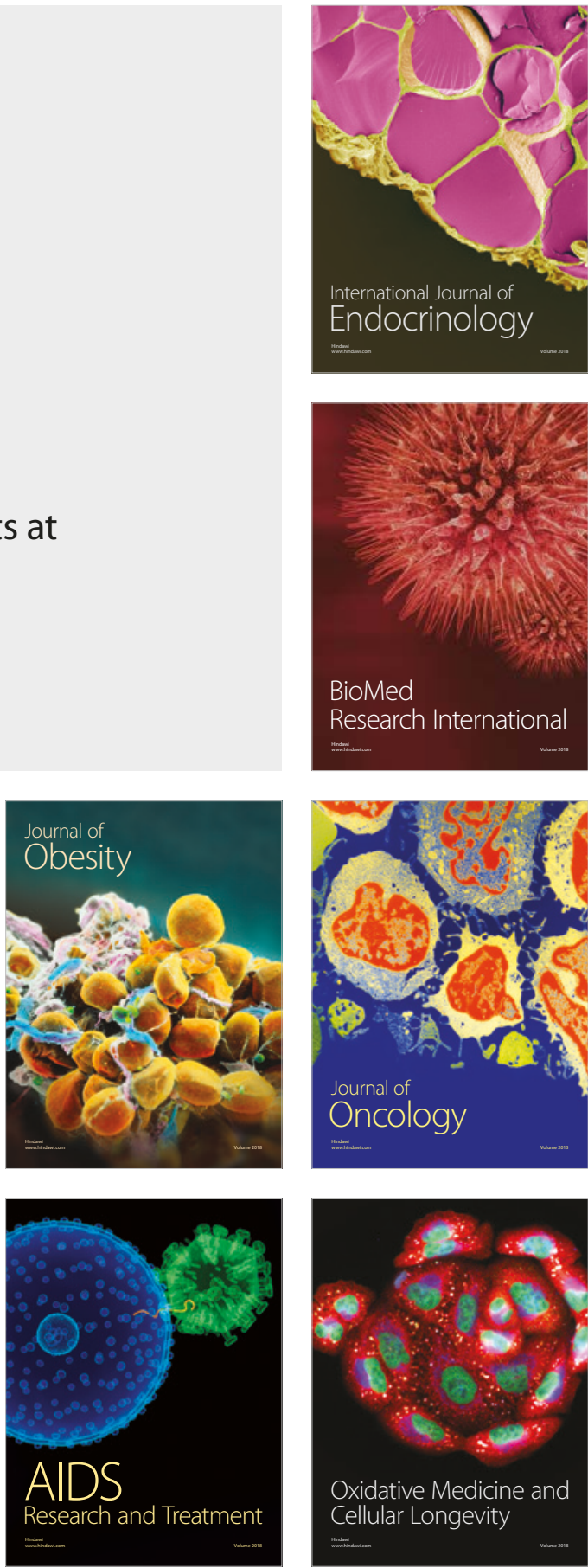